\title{
Subacute thyroiditis in a patient infected with SARS-COV-2: an endocrine complication linked to the COVID-19 pandemic
}

\author{
Rosaria Maddalena Ruggeri ${ }^{1}$ (D) $\cdot$ Alfredo Campennì $^{2} \cdot$ Massimiliano Siracusa $^{2} \cdot$ Giuseppe Frazzetto $^{3} \cdot$ Damiano Gullo $^{3}$
}

Received: 19 June 2020 / Accepted: 3 July 2020 / Published online: 16 July 2020

(C) Hellenic Endocrine Society 2020

\begin{abstract}
Purpose Subacute thyroiditis (SAT) is an inflammatory thyroid disorder of viral origin, generally preceded by an upper respiratory tract infection. Since the disorder is self-limiting, it is frequently underdiagnosed. However, the disease should not be overlooked since the associated thyrotoxicosis may worsen the clinical course of concomitant disorders (e.g., respiratory distress) and long-term sequelae, such as autoimmune hypothyroidism, have been reported.

Methods Here we describe a woman who developed SAT with thyrotoxicosis after SARS-COV-2 infection. Coronavirus disease (COVID-19) symptoms were mild and the patient was managed with no specific treatment and recovered rapidly.

Results Six weeks after the onset of the upper respiratory tract infection, the patient developed pain and tenderness in the anterior cervical region, fatigue, tremors, and palpitations. Physical examination revealed mild tremors of the extremities, a diffuse and painful goiter, and enlarged and tender cervical and submandibular lymph nodes. At biochemical evaluation, TSH was suppressed, FT3 and FT4 were high, and serum thyroglobulin was markedly increased (188 pg/mL; n.v. 0-40). Thyroid scintigraphy showed markedly reduced ${ }^{99 \mathrm{~m}} \mathrm{Tc}$-perthecnetate uptake in the gland. All findings were consistent with SAT, and treatment with oral prednisone $(25 \mathrm{mg} /$ day as the starting dose, gradually tapered) was started. Under the corticosteroid therapy, there was progressive resolution of symptoms and signs, and, within 4 weeks, all thyroid functional tests and inflammatory indexes normalized.
\end{abstract}

Conclusion Clinicians should be aware of thyroid manifestations potentially associated with COVID-19.

Keywords COVID-19; SARS-COV-2 · Subacute thyroiditis $\cdot$ Viral thyroiditis

Dear Editor,

Subacute thyroiditis (SAT) is a self-limiting inflammatory disorder of the thyroid and a relatively uncommon cause of thyrotoxicosis linked to a viral infection [1]. The acute onset of this disorder is often preceded by an upper respiratory tract infection caused by viruses such as influenza, adenovirus,

Rosaria Maddalena Ruggeri

rmruggeri@unime.it

1 Department of Clinical and Experimental Medicine, Unit of Endocrinology, "Gaetano Martino" University Hospital, University of Messina, 98125 Messina, Italy

2 Department of Biomedical Sciences and Morphological and Functional Images, Unit of Nuclear Medicine, University of Messina, Messina, Italy

3 Endocrine Unit, Department of Clinical and Molecular Biomedicine, Garibaldi-Nesima Hospital, University of Catania Medical School, Catania, Italy
Coxsackie, or, less frequently, Epstein-Barr and cytomegalovirus (CMV) [1]. Since the disorder is self-limiting, it is frequently misdiagnosed. The novel coronavirus, also known as severe acute respiratory syndrome coronavirus 2 (SARSCOV-2), is a RNA virus belonging to the coronaviridae family identified as the cause of severe acute respiratory syndrome, namely, coronavirus disease 2019 (COVID-19), declared as a pandemic by the World Health Organization in March 2020 [2]. We describe a case of SAT in a patient affected by COVID-19.

A 43-year-old woman with no personal history of thyroid disease developed fever, mild upper respiratory symptoms (i.e., rhinorrhea, painful swallowing, cough, and hoarseness), and conjunctivitis at the beginning of March 2020. Serum markers of acute inflammation were positive (erythrocyte sedimentation rate, $60 \mathrm{~mm} / \mathrm{h} ; \mathrm{C}$ reactive protein [CRP], $8.8 \mathrm{mg} / \mathrm{L}$ (normal, 0-5 mg/L), while white cell count was within the normal range $\left(6.6 \times 10^{9} / \mathrm{L}\right)$ with high lymphocyte percentage $(40 \%)$. Baseline chemistry and hepatic and renal function 
were all within normal ranges. The chest X-ray was normal. Oropharyngeal swab tests of SARS-COV-2 RNA were positive. The patient was managed with no specific treatment and recovered rapidly. Two further swabs for SARS-COV-2 14 and 28 days after the first one were negative. However, a lowgrade fever persisted, rising to $37.5^{\circ}$ in the evening. In midApril 2020, the patient suddenly developed pain and tenderness in the anterior cervical region, fatigue, tremors, and palpitations. The neck pain, which was severe and was aggravated by swallowing, was responsive to low-dose steroidal drugs (betamethasone $0.5 \mathrm{mg}$, twice a week) taken by the patient on her own initiative. Because of worsening of tremors, anxiety, and palpitations, thyroid function tests were requested by the general practitioner. Thyrotropin (TSH) was suppressed $(0.006 \mathrm{mU} / \mathrm{L}$; normal values $0.27-4.2)$; free triiodothyronine (FT3) was $7.03 \mathrm{pg} / \mathrm{mL}$ (n. v. 1.71-3.71); and free thyroxine (FT4) was $2.69 \mathrm{ng} / \mathrm{dL}$ (n. v. 0.7-1.48). Thyroglobulin (Tg) was markedly increased (188 pg/mL; n.v. 0-40), whereas anti-thyroglobulin antibodies ( $\mathrm{TgAb})$, anti-peroxidase antibodies (TPOAb), and anti-TSH receptor antibodies (TRAb) were undetectable. The patient was referred to the Endocrine Unit for further evaluation. Physical examination revealed mild tremors of the extremities, a diffuse and painful goiter, and enlarged and tender cervical and submandibular lymph nodes. The patient was found to have both IgM (40 IU = $\mathrm{mL}$; normal, $<15 \mathrm{IU}=\mathrm{mL})$ and $\mathrm{IgG}(0.73 \mathrm{IU}=\mathrm{mL}$; normal, $<0.4 \mathrm{IU}=\mathrm{mL}$ ) antibodies to SARS-COV-2, indicating that she had contracted the viral infection, which might have caused the thyroiditis. Neck ultrasonography showed a diffusely enlarged and hypoechogenic thyroid gland. Thyroid scintigraphy showed markedly reduced ${ }^{99 \mathrm{~m}} \mathrm{Tc}$-perthecnetate uptake in the gland (Fig. 1). All findings were consistent with SAT, and treatment with oral prednisone $(25 \mathrm{mg} /$ day as the starting dose, gradually tapered) was prescribed. Under the corticosteroid therapy, there was progressive resolution of symptoms and signs. Within 2 weeks, serum inflammatory indexes normalized and FT3 and FT4 decreased just above upper the normal range (FT4, $1.69 \mathrm{ng} / \mathrm{dL}$; FT3, $4.03 \mathrm{pg} / \mathrm{mL}$ ), while TSH was still low $(0.22 \mathrm{mUI} / \mathrm{L})$. Two weeks later, 4 weeks after starting steroid therapy, all thyroid functional tests normalized, as well as inflammatory indexes.

The COVID-19 pandemic started in December 2019 and spread worldwide, with over a million of people being infected over the following 6 months [2]. SARS-COV-2 exhibits marked tissue tropism causing mainly interstitial pneumonia and severe acute respiratory syndrome, inducing an aberrant inflammatory response that also involves the cardiovascular, coagulative, gastrointestinal, and nervous systems [2]. The whole spectrum of complications and long-term sequelae related to SARs-COV-2 infection is as yet far from being fully understood and, in particular, thyroid gland involvement is not yet clearly defined. Data from the related SARS-COV-1 showed that the virus was responsible for follicular cell destruction and dysfunction and fibrosis following the acute phase, representing the histopathological hallmarks of destructive thyroiditis [2].

We report a case of SARS-COV-2 associated with an infection of the thyroid gland. SAT occurred quite some time after resolution of upper respiratory manifestations. This, as well as two other cases of destructive thyroiditis associated with SARS-COV-2 infection that were almost simultaneously reported, highlight that potential thyroid involvement should be taken into account by physicians working in COVID-19 units [3]. If more cases occur, this disorder of the thyroid gland should be included as a possible complication triggered by SARS-COV-2 during the COVID-19 pandemic. Clinical suspicion of SAT should be kept in mind when assessing cases of COVID-19 in the presence of neck pain and symptoms of hyperthyroidism. Many patients infected with COVID-19 are not able to recall any known exposure or are

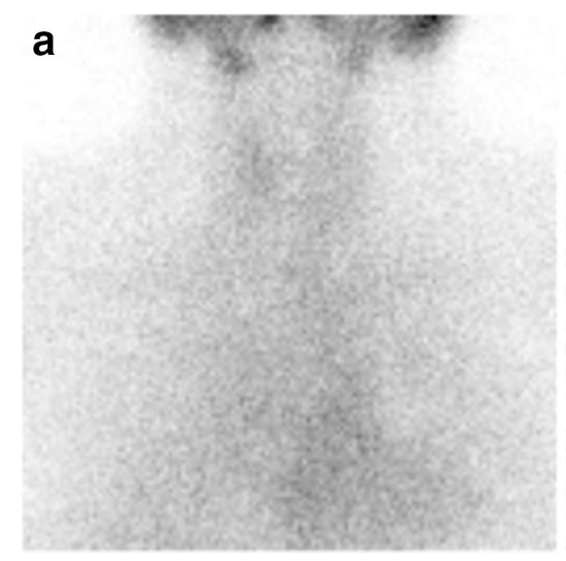

Fig. $1{ }^{99 \mathrm{~m}}$ Tc-pertechnetate scintigraphy obtained using a single-head gamma camera equipped with low-energy high-resolution parallel-hole collimator (LEHR-PAR). Panel A. Static image (magnification 1; matrix $256 \times 256$, acquisition frame $100 \mathrm{Kc}$ ) obtained in anterior view $10 \mathrm{~min}$

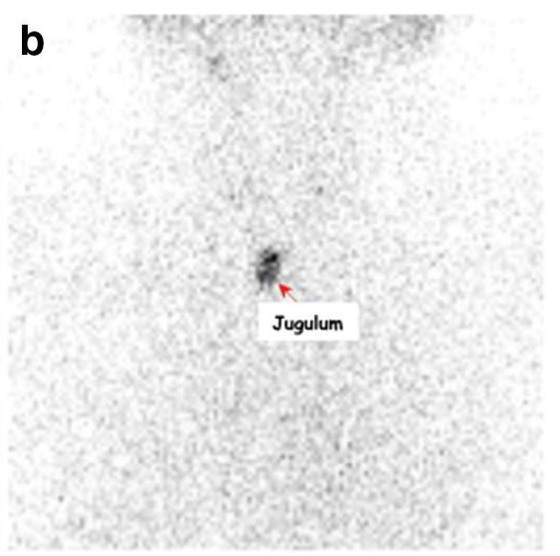

after radiotracer administration $(111 \mathrm{MBq})$. No significant ${ }^{99 \mathrm{~m}} \mathrm{Tc}$ pertechnetate uptake in thyroid parenchyma was noted. Panel B. To better identify the thyroid bed, a jugular radioactive mark was used 
asymptomatic. Confirming COVID-19 diagnosis may also significantly decrease the risk of disease transmission.

Contributions All authors made substantial contributions to the design, analysis, and interpretation of the data included and approved the final version for submission for publication. All authors agree to be accountable for the accuracy and integrity of the work.

Code availability Not applicable.

\section{Compliance with ethical standards}

Conflicts of interest The authors declare that they have no conflicts of interest.

Ethics approval/consent to participate/consent for publication The authors have no ethical conflict to disclose. Written informed consent was obtained from the patient for publication of this letter. Local Ethics Research Committee approval was obtained.

\section{References}

1. Desailloud R, Hober D (2009) Viruses and thyroiditis: an update. Virol J 6:5

2. Guan WJ, Ni ZY, Hu Y et al (2020) Clinical characteristics of coronavirus disease 2019 in China. N Engl J Med 382:1708-1720

3. Ippolito S, Dentali F, Tanda ML (2020) SARS-CoV-2: a potential trigger for subacute thyroiditis? Insights from a case report. J Endocrinol Invest. https://doi.org/10.1007/s40618-020-01312-7

Publisher's note Springer Nature remains neutral with regard to jurisdictional claims in published maps and institutional affiliations. 